\title{
Silicon sensitisation using light harvesting layers
}

\author{
Liping Fang ${ }^{1}$, Nicholas Alderman ${ }^{1}$, Lefteris Danos ${ }^{* 2}$ and Tom Markvart ${ }^{1}$ \\ ${ }^{1}$ Solar Energy Laboratory, Faculty of Engineering and the Environment, University of Southampton, \\ Highfield, Southampton, SO17 1BJ, UK \\ ${ }^{2}$ Department of Chemistry, Energy Lancaster, Lancaster University, Lancaster, LA1 4YB, UK \\ *Email: 1.danos@lancaster.ac.uk
}

\begin{abstract}
Langmuir Blodgett monolayers of a cyanine dye mixed with stearic acid were deposited on glass and silicon substrates with spacer layers of pure stearic acid monolayers or silicon dioxide films deposited by PECVD. By using the time correlated single photon counting technique, time-resolved emission spectra (TRES) and decay curves were measured to characterise the dependence of energy transfer rate on the separation between the dye monolayer and the silicon surface and also on the dye concentrations in the monolayers.

We observe interlayer energy transfer between monomers, dimers and higher aggregates present in the monolayer deposited on glass but also competing directly with energy transfer to silicon at close distances. We find that the fluorescence lifetime of the dye monolayer is significantly shortened when present close to the silicon surface signifying efficient energy transfer. The dissipation of the excitation energy near silicon is explained using the classical theory developed for metals and a deviation is observed for distances close to the silicon surface.
\end{abstract}




\section{Introduction}

Light harvesting layers in conjunction with thin silicon semiconductor converters represents an attractive solution for reducing the amount of semiconductor material needed by up to two orders of magnitude in the manufacture of solar cells. ${ }^{1,2}$ The idea of silicon sensitisation was first proposed by Dexter ${ }^{3}$ which postulated that electron-hole pair generation is possible via energy transfer from excited dye molecules on the silicon surface. An important feature of this energy transfer - which occurs from a localised electron state - is that it involves no strict momentum conservation.

The term "light harvesting" describes a process which enhances the absorption cross section of the photosynthetic reaction complex by excitation energy transfer, often called resonance energy transfer. ${ }^{4,5}$ This proposal opens the way for light harvesting and directed energy transfer for enhancement of the photo-excitation rate in indirect gap semiconductors. ${ }^{6,7}$ In application to crystalline silicon solar cells, light harvesting offers the promise of dramatic cost reduction by combining the advantages of high optical absorption of dye molecules with the efficient electronic properties of crystalline silicon. The key factor in designing successful light harvesting structures is the control of molecular organisation and inter molecular distances for a high efficiency of excitation energy transfer while avoiding other non-radiative dissipation processes (i.e. quenching). This requirement is successfully addressed by using Langmuir- Blodgett (LB) films which are shown to be well suited for this purpose. ${ }^{8}$

The modification of dye fluorescence near a metal interface has been discussed extensively in the literature, generally using a classical theory. ${ }^{9-11}$ The observed oscillations in the fluorescence lifetime at large distances to the metal surface were explained in terms of interference between the reflected wave and the initial wave. At a closer distance from the surface, the observed decrease in lifetime was attributed to nonradiative energy transfer from the excited dye molecule to the metal.

In contrast with fluorescence quenching near metals, not much is known about the relaxation pathways of molecules near the surface of a semiconductor. In the case of metal, the dipole-dipole type interaction between the excited dye molecule and the surface plasmon mode in the metal is described satisfactorily with the use of the (complex) dielectric function. The applicability of a similar model to semiconductor, however, is doubtful, especially close to the quenching region $(\mathrm{d}<10 \mathrm{~nm})$. This question remains open despite a number of experimental ${ }^{3,12-16}$ and theoretical ${ }^{17}$ studies. 
In this paper, we show results from LB dye layers deposited on glass and silicon surfaces at various distances and we also demonstrate efficient energy transfer (fluorescence quenching) from the dye overlayers to the silicon substrates. Sensitisation of silicon (or light harvesting), in this way would allow both the thickness of semiconductor required and the quality of semiconductor material to be reduced, as the majority of electron-hole pairs would be generated within a few nanometers of the surface. Crystalline silicon represents large proportion of the cost of a solar cell, and considerable savings can be made through a reduction in the material thickness. Changing the distance from the dye to silicon, would allow the dependence of this energy transfer from chromophore to semiconductor to be investigated. This can therefore help us to understand and improve upon the two principal functions of a solar cell (light capture and charge separation). By separating these functions, photovoltaic devices can be improved significantly. Concepts such as up-conversion ${ }^{18,19}$ and downshifting ${ }^{20,21}$ could also be included in future devices through this method, thereby enhancing their efficiencies.

\section{Experimental details}

\subsection{Materials and Methods}

The carbocyanine lipophilic dye 3,3' dioctadecyloxacarbocyanine perchlorate (DiO) was obtained from Invitrogen (Life Technologies). The rest of the chemicals stearic acid (SA), cadmium chloride, chloroform and hexamethyldisilazane (HMDS) were obtained from Sigma-Aldrich at the highest purity available and were used as received. Monocrystalline silicon wafers were obtained from Siltronix (100 orientation, P type, resistivity of 1-30 $\mathrm{ohm} \cdot \mathrm{cm}$ ). Standard microscopic glass slides were obtained from Corning. 


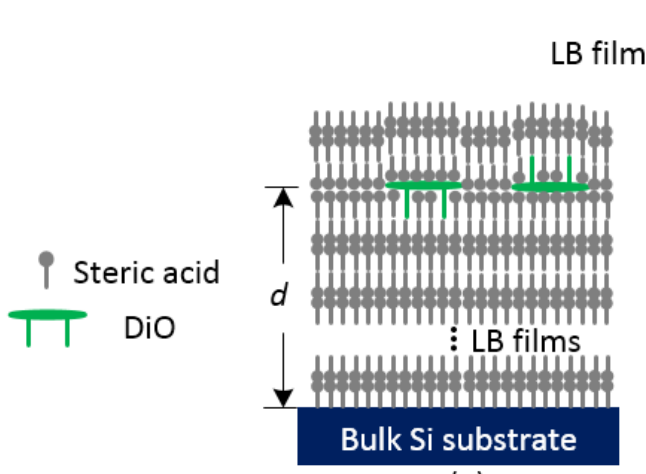

(a)

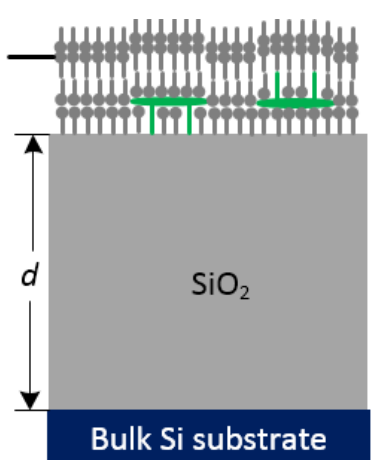

(b)

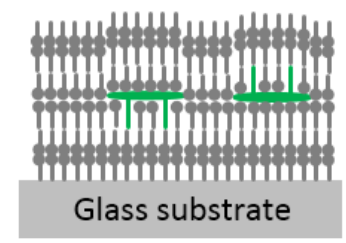

(c)

Figure 1. LB films deposited on silicon substrate with (a) stearic acid and (b) silicon dioxide as spacer layer and (c) on glass substrate.

Different mixing ratios of $\mathrm{DiO}$ and $\mathrm{SA}$ were prepared as solution in Chloroform. Langmuir monolayers were prepared in a Langmuir-Blodgett trough (KSV Nima Ltd) equipped with three dipping wells. LB Films were deposited as a Cadmium salt at a constant pressure of $28 \mathrm{mN} / \mathrm{m}$ and at a rate of $15 \mathrm{~mm} / \mathrm{min}$. Ultra pure water obtained from a Sartorius reverse osmosis system (ARIUM-UV) was used as the subphase with a resistivity of $18.2 \mathrm{Mohm} \cdot \mathrm{cm}$ and a total organic content (TOC) of less than $2 \mathrm{ppb}$. The subphase contained Cadmium Chloride (Aldrich) at a concentration $5 \times 10^{-4} \mathrm{M}$ and $\mathrm{pH}=5.6$.

Silicon dioxide of different thicknesses were deposited on silicon wafers by a PECVD system made by Oxford Instruments. The chamber was evacuated to 1000 mTorr before the introduction of silane, nitrogen and nitrous oxide. The ratio of these gases were well tuned to achieve the silicon dioxide deposition rate around $1 \mathrm{~nm} / \mathrm{s}$ with input $\mathrm{RF}$ frequency at $13.56 \mathrm{MHz}$ and power at $20 \mathrm{~W}$.

\subsection{Spectroscopic measurements and data analysis}

Fluorescence decay curves and time-resolved emission spectra (TRES) were obtained using time-correlated single photon counting (TCSPC). ${ }^{22}$ A FluoTime200 spectrometer (PicoQuant) equipped with a TimeHarp300 TCSPC board (PicoQuant) and a Hamamatsu photomultiplier (PMA-185) was used for all measurements. The excitation source was a $440 \mathrm{~nm}$ picosecond pulsed diode laser (PicoQuant, LDH440) driven by a PDL800-D driver (PicoQuant) operated at a variable pulse repetition rate $(10-40 \mathrm{MHz})$. The emission spectra were collected at right angles to the excitation wavelength and the emission pathlength was fitted with a long pass filter (HQ460LP, 
Chroma) before monochromator (Scientech 9030). The resolution of the system's instrument response function (IRF) had a full width half maximum (fwhm) of $350 \mathrm{ps.}$

The analysis of the fluorescence decay curves was carried out using the FluoFit software (PicoQuant, version 4.2.1) based on multi-exponential or stretched exponential models which involves an iterative reconvolution process. ${ }^{22}$ The time-resolved emission spectra (TRES) were measured from $480-750 \mathrm{~nm}$ with a $2 \mathrm{~nm}$ step. The steady state fluorescence spectra were constructed from the directly measured TRES spectra. ${ }^{22}$ The background emission from the bare glass substrate was subtracted and all emission spectra have been corrected against known emission standards. ${ }^{23}$ The thickness and the refractive index of the deposited silicon dioxide films and the LB films were measured in the wavelength range from 200-1700 $\mathrm{nm}$ via a J. A. Woollam Variable Angle Spectroscopic Ellipsometry (VASE) system in the cleanroom of Southampton Nanofabrication Centre.

\section{Results and Discussion}

\subsection{Orientation of DiO in the monolayer and previous studies}

According to previous pressure-area isotherm observations, ${ }^{24,8}$ the area occupied by a $\mathrm{DiO}$ molecule in the mixed monolayer on the water surface was estimated to be 0.45 $\mathrm{nm}^{2}$ and independent of the mixing ratio. This estimated area is smaller than the molecular cross section of the dye, indicating a mixing with the stearic acid at the molecular level where the stearic acid chain is inserted in between the octadecyl chains in the $\mathrm{DiO}$ molecule. The molecular area of the pure dye monolayer was found to be $0.65 \mathrm{~nm}^{2}$ which is larger than the dye area in the mixed monolayers and could indicate edge on orientation of the molecule on the monolayer where the molecule lies flat on the plane of the monolayer. Polarisation measurements of the absorption spectrum have indicated the molecule to lay nearly flat on glass substrates having a random orientation of their long axes with the transition dipole moment orientation. ${ }^{24,8}$ The measured lifetime results (intensity averaged) (see section 3.4) fit better with the modelling results of a horizontal electric dipole (HED), and the unquenched lifetime obtained from this fitting $(1.25 \mathrm{~ns})$ is closer to the intensity averaged lifetime measured from glass sample 
(1.3 ns), than the unquenched lifetime obtained from fitting with either a vertical electric dipole (VED) or an isotropic configuration of dipole orientation. ${ }^{9}$

\subsection{Steady State Emission Spectra constructed from Time Resolved Emission Spectra (TRES)}

The normal steady state fluorescence spectra can be constructed from the TRES spectra by integrating the time resolved spectra over the whole time region of acquisition. ${ }^{22,25}$ The spectra presented in this section have been constructed from the direct recording of TRES spectra. The background emission from the bare glass substrate has been subtracted for all spectra.

The fluorescence spectra of DiO mixed with SA in LB monolayers deposited on glass substrates with mixing ratios of 1:1000 up to a 1:1 monolayer are shown in Figure 2. The spectra for 1:1000 mixing ratio are similar to the one of $\mathrm{DiO}$ in $\mathrm{CHCl}_{3}$ for low concentration $\left(10^{-7} \mathrm{M}\right)$ with an emission maximum at $\sim 500 \mathrm{~nm}$. As the dye concentration is increased to $1: 100$ there is a broadening of the emission band. At further increase of the mixing ratio (1:50) there is a shift of the maximum at $540 \mathrm{~nm}$ and a new band appearing at $580 \mathrm{~nm}$. At even higher dye concentrations the $500 \mathrm{~nm}$ peak is significant decreased and peaks appear at $546 \mathrm{~nm}$ and $582 \mathrm{~nm}$. There is a broadening of the emission band at higher mixing ratios accompanied by a small shift of the emission peak at $590 \mathrm{~nm}$ together with a decrease of the $546 \mathrm{~nm}$ peak. The emission at a mixing ratio of $1: 1$ is evident of the almost complete disappearance of the $500 \mathrm{~nm}$ peak, the broaden feature of the $590 \mathrm{~nm}$ peak which is now the maximum peak and the decrease of the $546 \mathrm{~nm}$ peak. 


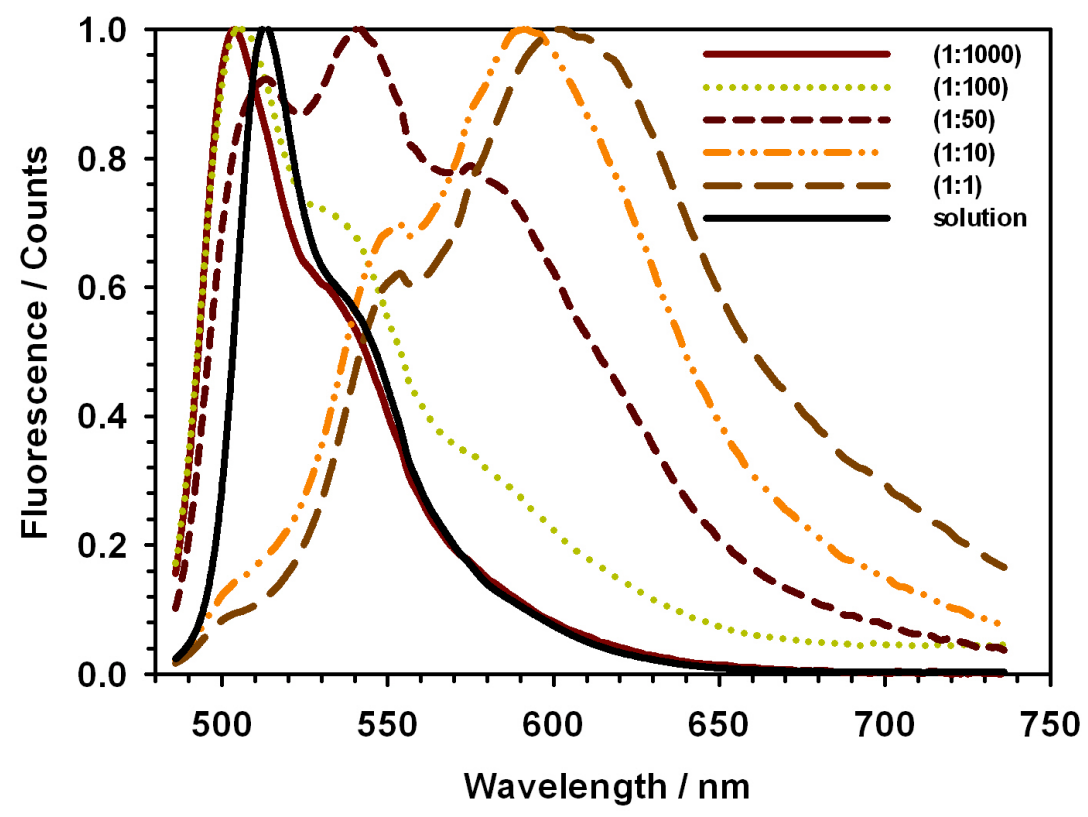

Figure 2. Emission spectra recorded directly from TRES spectra for DiO:SA LB monolayers on glass for different mixing ratios. The emission spectra for $\mathrm{DiO}$ in Chloroform solution $\left(\mathrm{C}=10^{-7} \mathrm{M}\right)$ are also shown for comparison.

\subsection{Time resolved Emission Spectra (TRES)}

Normalised TRES spectra are shown in Figure 3 for concentrations ranging from 0.01$50 \mathrm{~mol} \%$ (mixing ratios $1: 1000$ to $1: 1$ ). The spectra can also be deconvoluted into individual contributions from the monomer, dimer and higher aggregates present in the monolayer occurring at increased dye concentrations. ${ }^{7}$ For the spectra $0.01-0.1 \mathrm{~mol} \%$ (mixing ratios $1: 10000$ to $1: 1000$, only $1: 1000$ shown here)) there is nearly no change observed on the emission spectrum during the time region (0-25 ns) monitored and these spectra correspond to the monomer emission.

At concentrations greater than $1 \mathrm{~mol} \%$ (mixing ratios above 1:100) the following observations can be made: 
(1:100)- The spectra initially are similar in shape to the monomer spectrum, with the passage of time there is a broadening of the emission band and peaks appear at $514 \mathrm{~nm}$, $540 \mathrm{~nm}$ and $580 \mathrm{~nm}$ while the peak at $500 \mathrm{~nm}$ decreases.

(1-50)- The spectra appear to be broader initially indicating the appearance of dimers in the LB film, with the passage of time a peak appears at $514 \mathrm{~nm}$ and the $500 \mathrm{~nm}$ peak decreases and later two peaks appear at $540 \mathrm{~nm}$ and $580 \mathrm{~nm}$

(1:10)- The monomer peak at $500 \mathrm{~nm}$ is not strong indicating the disappearance of monomers in the LB Film. The peaks at $545 \mathrm{~nm}$ and $580 \mathrm{~nm}$ appear in the beginning indicating direct photon excitation of dimer and higher aggregate. With the passage of time there is further broadening and a gradual shift of the $580 \mathrm{~nm}$ peak indicating the formation of higher aggregates. The peak at $540 \mathrm{~nm}$ decreases and the peak at $580 \mathrm{~nm}$ increases (always with respect to each other)

(1:1)- There is a small monomer contribution to the spectrum. Peaks at $540 \mathrm{~nm}$ and 580 $\mathrm{nm}$ appear immediately after photoexcitation indicating the appearance of dimers and higher aggregate in the film. The broadening and shifting of the $580 \mathrm{~nm}$ peak is more pronounced and the decrease of the $540 \mathrm{~nm}$ peak more pronounced as well, indicating energy transfer from the dimer and to higher aggregates in the film.

The following class of relaxation pathways exist in the system of $\mathrm{DiO}$ deposited on glass:

\section{(i) Direct photoexcitation}

$$
\begin{aligned}
& \mathrm{M}+\mathrm{hv} \rightarrow \mathrm{M}^{*}(1-1) \\
& \mathrm{D}+\mathrm{hv} \rightarrow \mathrm{D}^{*}(1-2) \\
& \mathrm{H}+\mathrm{hv} \rightarrow \mathrm{H}^{*}(1-3)
\end{aligned}
$$

(ii) Energy Migration (donor to donor ET)

$$
\begin{aligned}
& \mathrm{M}^{*}+\mathrm{M} \rightarrow \mathrm{M}+\mathrm{M}^{*}(2-1) \\
& \mathrm{D}^{*}+\mathrm{D} \rightarrow \mathrm{D}+\mathrm{D}^{*}(2-2) \\
& \mathrm{H}^{*}+\mathrm{H} \rightarrow \mathrm{H}+\mathrm{H}^{*}(2-3)
\end{aligned}
$$

(iii) Energy Transfer

$$
\begin{aligned}
& \mathrm{M}^{*}+\mathrm{D} \rightarrow \mathrm{M}+\mathrm{D}^{*}(3-1) \\
& \mathrm{D}^{*}+\mathrm{H} \rightarrow \mathrm{D}+\mathrm{H}^{*}(3-2) \\
& \mathrm{M}^{*}+\mathrm{H} \rightarrow \mathrm{M}+\mathrm{H}^{*}(3-3)
\end{aligned}
$$

where $\mathrm{M}, \mathrm{D}, \mathrm{H}$ are monomer, dimer and higher aggregate respectively. 
For the discussion of the relaxation pathways of $\mathrm{DiO}$ in mixed stearic films the mixing ratios can be divided into roughly three regions.

\section{Region 1 (low dye concentrations, mixing ratios 1:10000, 1:1000)}

The monomers are only present in the monolayer and they get photoexcited directly But also, we have energy migration (donor-donor energy transfer) until the excitation energy reached an energy trap. The nonexponential decay of the lowest mixing ratios of $\mathrm{DiO}$ could indicate this efficient energy migration from monomer to monomer but can also be because of the non-uniform environment around the dye molecules from the stearic acid.

\section{Region 2 (medium dye concentrations, mixing ratios 1:100 until 1:50)}

In addition to direct photoexcitation of the monomers we have a small proportion of dimers in the layer which get photoexcited via energy transfer from the monomer. This pathway is more significant and that is why we observe the appearance of the peaks at a later time in the emission process.

\section{Region 3 (high dye concentrations, mixing ratios above 1:50)}

In this system, there is a competition between direct photoexcitation of dimers and excitation via energy transfer from the monomers. As we increase the concentration, the monomer concentration formed in the layer drops significantly in favour of dimer and higher aggregate formation, The spectra show two peaks at $540 \mathrm{~nm}$ and $580 \mathrm{~nm}$ which can indicate the emission from dimer (D) and higher aggregate (H1). These species can get photoexcited directly, but also via energy transfer from monomers or in the case of $\mathrm{H} 1$ from the dimer. It appears the two peaks at $540 \mathrm{~nm}$ and $580 \mathrm{~nm}$ to appear together on the onset of the excitation signifying the formation of dimer and higher aggregates in the monolayer. The emission peak of the monomer at $500 \mathrm{~nm}$ is negligible indicating the presence of very few monomers at these high dye concentrations. In this region we observe the energy transfer from the dimer to the higher aggregate and the broadening of the peak while the peak at $540 \mathrm{~nm}$ decreases. 

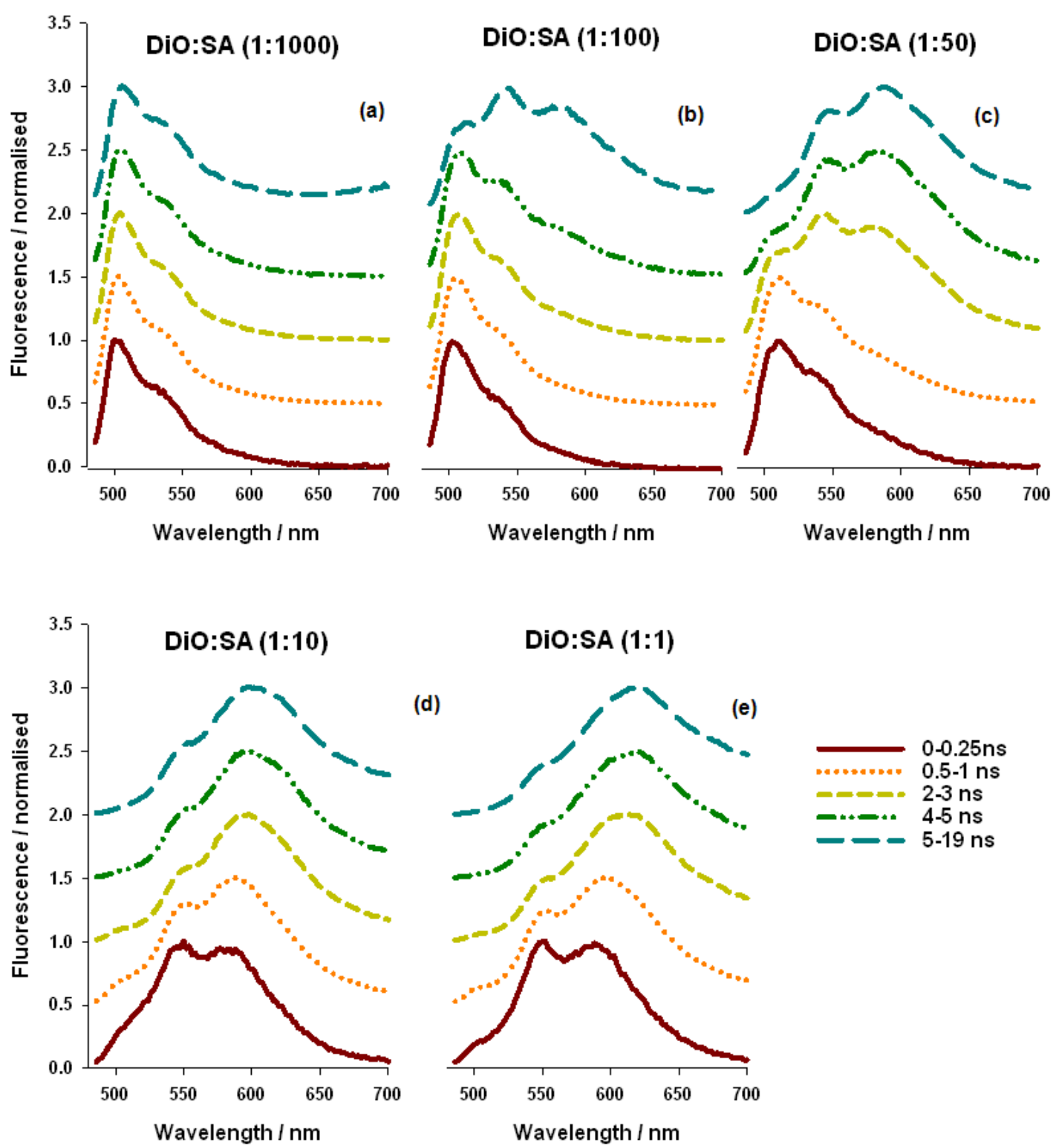

Figure 3- Time-resolved emission spectra of DiO in LB films at mixing ratios with SA of (a) 1:1000, (b) 1:100, (c) 1:50, (d) 1:10 and (e) 1:1. The excitation wavelength is $440 \mathrm{~nm}$.

\subsection{Fluorescence quenching on silicon substrates}

DiO:SA monolayers were deposited at different distance to the surface of bulk silicon substrates. For short distance pure SA LB films were used as a spacer layer, while for large distances, $\mathrm{SiO}_{2}$ films deposited by PECVD were used as spacer. The structure of these two kinds of samples is shown in Figure 1. The power dissipated by the excited molecules near a bulk silicon substrate can be broadly distinguished into two 
channels: fluorescence in the far field and light harvesting via energy transfer in the near field.

We have carried out a series of experiments where we measure the fluorescence lifetime of LB dye monolayers as a function distance to silicon. As shown in Figure 4, the measured results are fitted to the model of Chance-Prock-Silbey (CPS) [9]. The model appears to work really well for distances greater than $50 \mathrm{~nm}$ (far field) but shows apparent failings close to the silicon surface (near filed). At a close distance from the silicon surface the CPS model gives the lifetime $\tau_{\mathrm{d}}$ as proportional to $\mathrm{d}^{3}$, with the constant of proportionality determined by the imaginary part of the dielectric constant. This model was developed for metals and even when applied with silicon parameters, some disagreement from observation in the current situation is expected. We are currently developing a modified model to describe the observed discrepancy of the experimental results and it will appear in a separate publication.

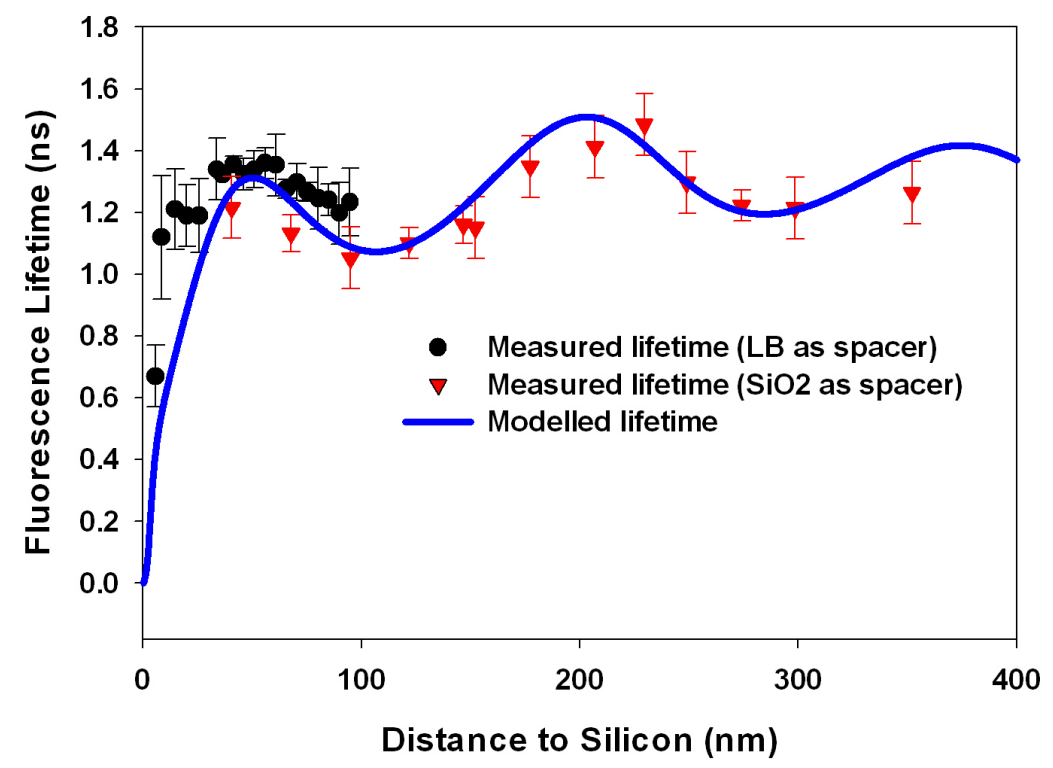

Figure 4. Measured fluorescence lifetimes for DiO:SA (1:100) at different distances to the silicon surface. 


\section{Conclusions}

The mixture of DiO and SA molecules were deposited as Langmuir-Blodgett monolayers on glass and silicon substrates. By changing the mixture ratio of $\mathrm{DiO}$ and SA molecules from 1:1000 to $1: 1$, monomer emission was observed at low concentrations and two broad bands assigned to two types of aggregates (dimer (D) and higher aggregates $(\mathrm{H}))$ were observed at higher concentrations. The band formation and contribution to the emission spectra depends strongly on the concentration of the $\mathrm{DiO}$ chromophores in the monolayer. The various energy transfer processes after photoexcitation has been discussed using time resolved emission spectra (TRES).

A series of LB film spacer structures were fabricated on top of silicon to control the distance of the overlying dye monolayer to the silicon surface. The fluorescence lifetime were measured at each step and it was found that the fluorescence emission was quenched close to the silicon surface. The measured fluorescence lifetime results were compared to a classic model and good agreement was found at the far field (d $>50 \mathrm{~nm})$ but the model was not able to describe the fluorescence quenching observed at shorter distances (Near Field). 


\section{References}

1. T. Markvart, Prog. Quantum Electron., 2000, 24, 107-186.

2. T. Markvart, L. Danos, N. Alderman, L. Fang, and T. Parel, in 27th European Photovoltaic Solar Energy Conference, Frankfurt, 2012, pp. 1-6.

3. D. L. Dexter, J. Lumin., 1979, 18/19, 779.

4. T. Förster, Ann. Phys., 1948, 2, 55.

5. D. L. Andrews, Ed., Energy Harvesting Materials, World Scientific Publishing Co. Pte. Ltd, 2005.

6. L. Danos, R. Greef, and T. Markvart, Thin Solid Films, 2008, 516, 7251-7255.

7. L. Danos and T. Markvart, Chem. Phys. Lett., 2010, 490, 194-199.

8. H. Kuhn, D. Mobius, and H. Bucher, in Techniques of Chemistry, eds. A. Weisberger and B. Rossiter, Wiley, New York, 1972, pp. 577-702.

9. R. . Chance, A. Prock, and R. Silbey, Adv. Chem. Phys., 1978, 65, 1.

10. H. Kuhn, J. Chem. Phys., 1970, 53, 101.

11. W. L. Barnes, Components, 1998, 45, 661-699.

12. T. Hayashi, T. G. Castner, and R. W. Boyd, Chem. Phys. Lett., 1983, 94, 461.

13. P. M. Whitmore, A. P. Alivisatos, and C. B. Harris, Phys. Rev. Lett., 1983, 50, 1092.

14. A. P. Alivisatos, M. F. Arndt, S. Efrima, D. H. Waldeck, C. B. Harris, and A. A. P., J. Chem. Phys., 1987, 86, 6540.

15. A. G. Vitukhnovsky, M. I. Sluch, A. G. Vitukhnosky, and M. C. Petty, Phys. Lett. A, 1995, 200, 61.

16. S. Huber and G. Calzaferri, Chemphyschem, 2004, 5, 239-42.

17. M. Stavola, D. Dexter, and R. Knox, Phys. Rev. B. Condens. Matter, 1985, 31, $2277-2289$.

18. a Shalav, B. Richards, and M. Green, Sol. Energy Mater. Sol. Cells, 2007, 91, 829-842.

19. S. Baluschev, T. Miteva, V. Yakutkin, G. Nelles, a. Yasuda, and G. Wegner, Phys. Rev. Lett., 2006, 97, 7-9. 
20. E. Klampaftis, D. Ross, K. R. McIntosh, and B. S. Richards, Sol. Energy Mater. Sol. Cells, 2009, 93, 1182-1194.

21. L. Danos, T. Parel, T. Markvart, V. Barrioz, W. S. M. Brooks, and S. J. C. Irvine, Sol. Energy Mater. Sol. Cells, 2012, 98, 486-490.

22. D. V. O'Connor and D. Phillips, Time-correlated single photon counting, Academic Press, London, 1984.

23. J. A. Gardecki and M. Maroncelli, Appl. Spectrosc., 1998, 52.

24. G. Biesmans, M. Van Der Auweraer, and F. C. De Schryver, Langmuir, 1990, 6, 277-285.

25. J. R. Lakowicz, Principles of Fluorescence Spectroscopy, Springer, New York, 3rd editio., 2006. 